\title{
Preoperative versus intraoperative endoscopic sphincterotomy for management of common bile duct stones
}

\author{
Manojkumar S. Nair
}

Published online: 27 February 2011

(C) Springer Science+Business Media, LLC 2011

We read with special interest the article, Preoperative Versus Intraoperative Endoscopic Sphincterotomy for Management of Common Bile Duct Stones, by El Geidie and colleagues published online in Surgical Endoscopy 17 September 2010. The authors should be commended for this well-designed trial. A large well-selected exclusion criteria identified uncomplicated symptomatic gallbladder disease with or without common bile duct (CBD) stones.

A few questions to the authors will be of interest to the readers. The method of randomization used was not mentioned. Also, the timing for opening of the concealed envelope and who was responsible for opening the envelope are not clear. These questions are important for readers, who need to know whether an allocation bias crept into the study.

One patient in the laparoscopic cholecystectomy (LC)/ IOES group had wound infection. What was the antibiotic policy used in this trial? Also, did the patient undergo open cholecystectomy (OC) or LC?
Failed stone extraction occurred for $3.2 \%$ the patients in the preoperative endoscopic sphincterotomy (PES)/LC group, and they were treated by endoscopic stent insertion followed by OC and CBD exploration. Technically, these patients underwent OC and are outside the perview of LC. In this context, it is interesting to recall recent evidence suggesting that CBD duct stenting can make subsequent surgery demanding [1]. One patient in the PES/LC group had bile leak. Was this a stented OC patient?

Disclosures Manojkumar S. Nair has no conflicts of interest or financial ties to disclose.

\section{Reference}

1. Nair MS, Uzzaman MM, Fafemi O, Athow A (2011) Elective laparoscopic cholecystectomy in the presence of common bile duct stent. Surg Endosc 25(2):429-436

M. S. Nair $(\bowtie)$

Department of General Surgery, North Middlesex University

Hospital, London N18 1QX, UK

e-mail: nair.manoj.dr@googlemail.com 